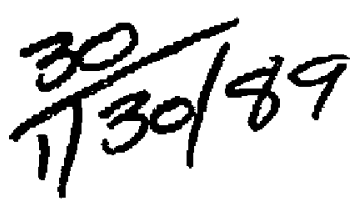

$\operatorname{CONF}-8.0062=$

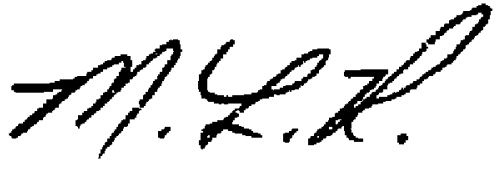

SLAC-POB- -4768

DE89 006ก66

\title{
THE STRANGEONIUM SPECTRUM SEEN IN LASS: IMPLICATIONS FOR GLUEBALL SPECTROSCOPY
}

D. Aston,' N. Awzji, 'T. Bienz,' F. Bird,' I. D'Amore, ${ }^{3}$ W. Dunwoodie,'

R. Endorf, ${ }^{3}$ K. Fujiit, ${ }^{2}$ H. Hayashii, ${ }^{2}$ S. Jwata, ${ }^{2}$ W.B. Johnson, ' R. Kajikawa, ${ }^{2}$

P. Kunz., D.W.G.S. Leith,' L. Levinson,' T. Matsui, ${ }^{2}$ B.T. Meadows, ${ }^{3}$

A. Miyamoto. ${ }^{2}$ M. Nussbaum, ${ }^{3}$ H. Ozaki, ${ }^{2}$ C.O. Pak, ${ }^{2}$ B.N. Ratcliff, ${ }^{2}$ D. Schuliz,

S. Shapiro.' T. Shinomun, 2 P. K. Sinervo,' A. Sugiyama., S, Suzuki, ${ }^{2}$

G. Tymopolsky, ' T. Tauchi, ${ }^{2}$ N. Toge, ${ }^{1}$ K. Ukri, ${ }^{4}$ A. Waite,' ${ }^{2}$ S. Willians'

1Stanford Linear Accelerator Center, Stanford University, Stanford, California 94309

2Nagoya Universiny. Furo-cho, Chikusa-ku, Nagoya 464, Japan

University of Curcimati, Cincinnari, Ohio 4522I

Insriture for Nuclear Sady, Universiny of Tokyo, Midori-cho. Tokyo I88, Japan

\section{ABSTRACT}

The stalus of strangeonium spectroscopy is re-assessed following our recent high satistics study of $\mathbf{K}$-induced hypertharge exchange reactions. The implications of our results for the status of glueball, or otherwise exotic, candidates observed in the sure decay modes but produced by different mechanisms are also discussed.

\section{INTRODUCTION}

As rocently as $19866^{\prime}$ only the $\eta / \eta^{\prime} . \phi(1020)$ and $f_{2}^{\prime}(1525)$ were clearly established as $\mathbf{s}$ members of complexe meson nonets predicted by the quark model. The \$(1850) was a good candidare for the 3- nonet, although us spin was untetermined. Winh the exctpion of the ground state $\eta m^{\prime}$, calculation of the octet-singlet mixing angles gives values elose to the magic value of $35^{\circ}$, so that these states have almost pure 5 quark content. The $f_{1}(1420)$, or $E$ meson, had been assigned to the $\mathrm{PC}_{=1}{ }^{++}$nonet. although there was considerable cooubt about its spin-parity

\section{EXPERIMENTAL DETALLS}

This ulk reviews the analyses of three hypercharge exchange reactions from a 4,I event/nb sample of I I GeV/c K p interactions detected in the LASS spectrometer a SLAC. ${ }^{2}$ The spectromeler is siluated in a clean RF separated beam, has an efficient trigger for final stares with $\geq 2$ charged particles and a flat acceptance over the full solid

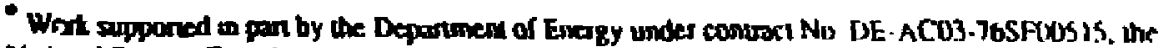
Narional Science Fautidation under gan Nas. PHYB2-09144, PHYR5-1380Bs. and the Japats L.\$. Cocperative Reserch Projea oo High Energy Physucs

Inviled talk presented by D. Ashu at the BNL Workshop on Clueballs, Hybrids, and Exotic Hadrons, Augusr 29-Septersber 1. 1988
} 
angie. Efficient unck-finding and topology re'onstruction combined with good panicle identification and application of geomerric and kinematic constraints at all decay vertices enabled us to find clean data samples of the reactions:

$$
\begin{aligned}
& K^{*} \mathrm{p} \rightarrow K^{-} K^{+} \Lambda \\
& K^{-} \mathrm{p} \rightarrow K_{\xi}^{0} K_{\xi}^{\circ} \Lambda \\
& K^{*} \mathrm{P} \rightarrow K^{\mp} K_{\xi}^{0} \pi^{ \pm} \Lambda
\end{aligned}
$$

In each case, all $V^{\circ}$ decay products are fully reconstructed. All these final states are dominated by hypercharge exchange production of a forward meson system at smal! momentum transfer $t^{\prime} \equiv 1:-t_{\text {mur }}$ from the incident beam. Space constraints permit only a brief description of the analyses; furher delails can be found in Refs 3-4, 5 and 6 for reactions (1), (2) and (3) respectively, and in the balk of Blair Ratcliff 7 elsewhere in these proceedings.

\section{$s \overline{\mathbf{s}}$ RESULTS}

The production mechanism is extremely important for selectung sis states; the meson funal state is nol necessarily a good indicator. For examplc, the $K^{-} K^{+}$spectrum produced in reaction (1) is totally different from that produced at a sinilar energy by charge exchange with a $\pi^{-}$beam 8 . Hypercharge exchange produces dominantly $1=0$ $\$ \overline{5}$ states in reactions $(1-3)$.

Figure 1 shows the $\bar{K} K$ specua from reactions (1) and (2): the $f_{2}^{\prime}(1525$ ) is clearly seen in boib reaclions and dominates the $K_{5}^{0} K_{5}^{0}$ spectrum Sinci: there is no Bose-Einstein restriction to even spin, the $K^{-} K^{+}$specirum also shows a large $\phi(1020)$ and evidence of the $\phi_{1}(1850)$. The corresponding Daliz plots (Fig 2) show that the continuum in $\mathrm{K}^{-} \mathrm{K}^{+}$above $-1,7 \mathrm{GeV} / \mathrm{c}^{2}$ is largely due to diffractive production of $\mathrm{N}^{*} \rightarrow \mathrm{K}^{+} \Lambda$. As expected, there is no evidence of sucli a mechanism in Fig. $2 b$.

Figure 3 shows the results of a spherical harmonic moments analysis in the Got. tried-Jackson frame of the low $t^{\prime}$ data from reaction (1) above the $f_{2}^{\prime}(1525)$. The peak at $-1.85 \mathrm{GeV} / \mathrm{c}^{2}$ in the moments up to $L=6$, but not higher, clearly denor.strates that the $\phi_{\mathrm{J}}(1850)$ has spin 3 . There is also some activily around $2.2 \mathrm{GeV} / \mathrm{c}^{2}$ in all moments up to $L=8$; monnents with $L>B$ (not shown) are censistert with zero $A$ complete amplitude andysis of the $Q_{3}(1850)$ region at both low and high $t$ shows that these dalia are consistent with a simple model where the leading $F$-wave interferes with an imaginary diffractive background as : 1 ggested by the Dalız plut. Figure 4 shows lin. eas combinations of the mornents which in this model are dominated by interierences between the leading F-wave and S, P and $D$ amplitudes. The superimposed curves are a fit to the mode! which gives $\$ 3(1850)$ parameters consisten with fits 10 in and the $F$. wave exuacted from the amplitude analysis (see Fig. 13 of Kef. 7).

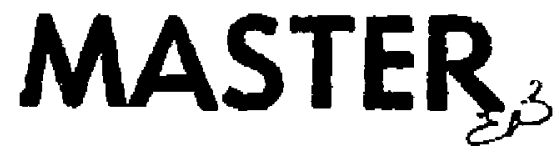



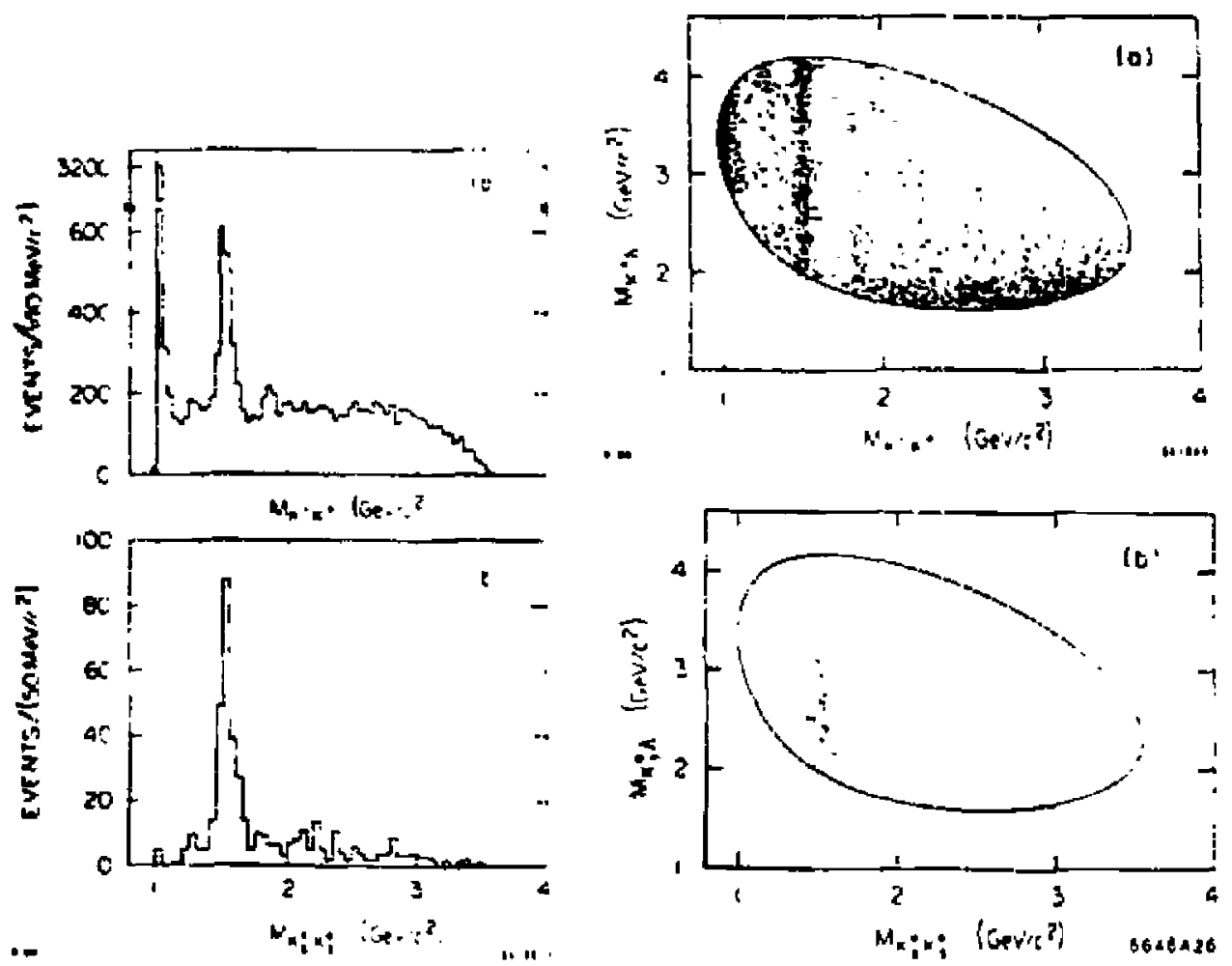

Fig. 1. The $\bar{K} K$ mass spectra from (a) reaction (1) and (b) reaction (2).

Fig. 2. The Dalitz plots from (a) reaction (1) and (b) reaccion (2).

We have exiended this model to the high mass data and find that the effect at 22 GeV/c $\mathrm{c}^{2}$, as suggested by the vanushing $\mathrm{l} .>8$ mornents, is consistent with a leading G-wave inlerfering with a similar background.

Figure 5 shows the constructive interference terms with $S, P, D$ and $F$ amplirudes: their sum (Fig. 14 of Ref. 7) shows a signal in the $2.16-2.28 \mathrm{GeV} / \mathrm{c}^{2}$ range with significance -30 , while the $t_{9}^{0}$ and 18 moments differ from zero by 33 and 2.30 respecovely over the same range Such a $\mathrm{JPC}=4^{++}$state, the ril 2210 , has mass and wndth consistent with those of the $X(222(1)$ seen by the Mark 111 collaturation $y$ and fits well as the sī member of the $4^{+}$nones predicted by the quark model It should atlus be noted that our data from reacuon ( 2 i in this mass region are consistent $w$ th the $\mathrm{K}_{\xi}^{\circ} \mathrm{K}_{5}^{\circ}$ specoum seen by the Mark III and IM2 collaborations in radiauve I/ $\Psi$ decay

A similar comparison of the low mass spectrum from redstuon (2) shows the strikung absence of the $f_{2}(1720)$ from our data, strongly suggesting that this state is

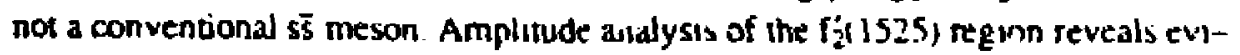




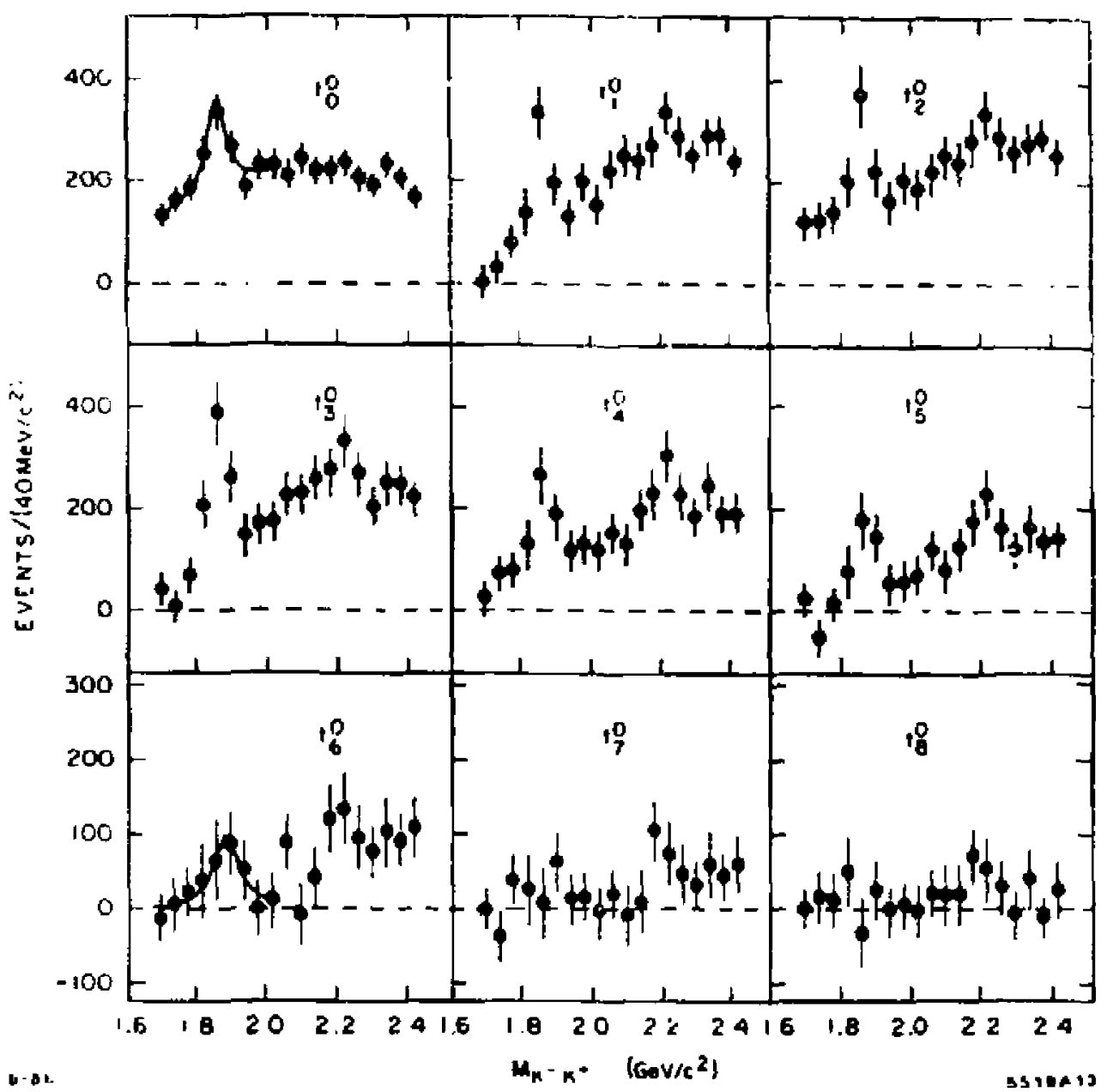

Fig. 3. The unnormalised $M=0 \quad \bar{K} K$ moments from reaction (1) above the $f_{2}^{\prime}(1525)$ with $1^{\prime}<0.2(\mathrm{GeV} / \mathrm{c})^{2}$ required.

dence for an underlying S-wave, shown in Fib. 12a of Ref. 7 which, though poorly measured, is significant at the 50 level and is produced with a cross-section consustem with expeciation for the underlying $s \bar{s} 0^{++}$onplet state Findly, the threshoid regiki shown in Fig. 6 has a surprising cluster of six events beluw $1.03 \mathrm{GeV} / \mathrm{c}^{2}$. Although not inconsisten with the $f_{0}(975)$, the pole on the real axis inferred by $A \cup$ el al 10 from the $\bar{K} K$ and $\pi \pi S$-wave scaltenng data provides a better descripoon of these events at the $90 \%$ confidence level.

Figure 7 shows the total $\bar{K} K \pi$ mass specorum from reactions (3). Its dominamt features are a sharp rise at $K * K$ threshold and an enhancement in the region of the $f_{1}(1530) 11$ There is litue evidence of the $f_{1}(1420)$ or the $\eta(1440)$, and just a feu 


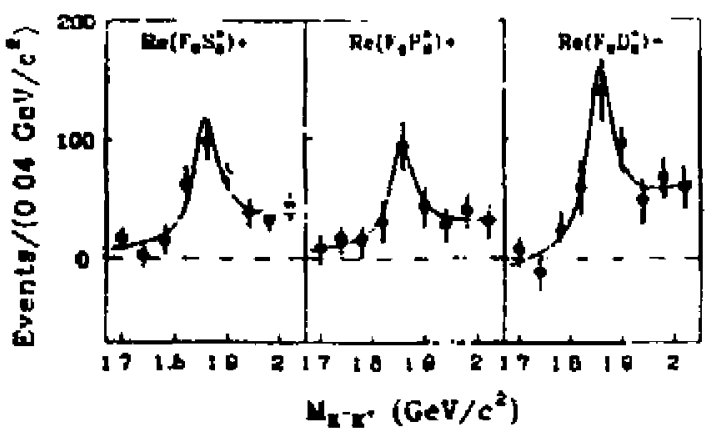

Fig. 4. Moments sums from teaction (1) dominated by leading $F$-wave interference terms according to the model described in the text.

events consistemt with the $f_{1}(1285)$. A three hody PWA reveals that production of $J P=1^{t}$ $\mathrm{K}^{*}$ waves dominates below $1.6 \mathrm{GeV} / \mathrm{c}^{2}$. There is evidence of interference belween the $K$ * bands which begins destructively at $K^{*} K$ threshold, but becomes constructive by -1.46 $\mathrm{GeV} / \mathrm{c}^{2}$. This effect is seen quite clearly in the Doliz plots of Fig. 8 and was also present in the data of Ref. 11. By combining the $1^{+}$ waves into G-parity eigenstates (see Fig. 15 bc of Res 7) and using the fact that $\mathrm{C}=\mathrm{G}$ for $I=0$ stan:s. we unfer the existence of a $J^{P C}=1^{+-} s \bar{s}$ state, the hí(1380), right at $K * K$ threshold and confirm the $\mathrm{I}^{++} \mathrm{ff}$ (1530). These states complete the two $1^{+}$nonets expected in the quark model and are consistent with having large $s \bar{s}$ content. It is intriguing that if we then assume magic mixing for these nonets, the calculaled masses of the $K_{1}$ states are very close to the physically observed masses.

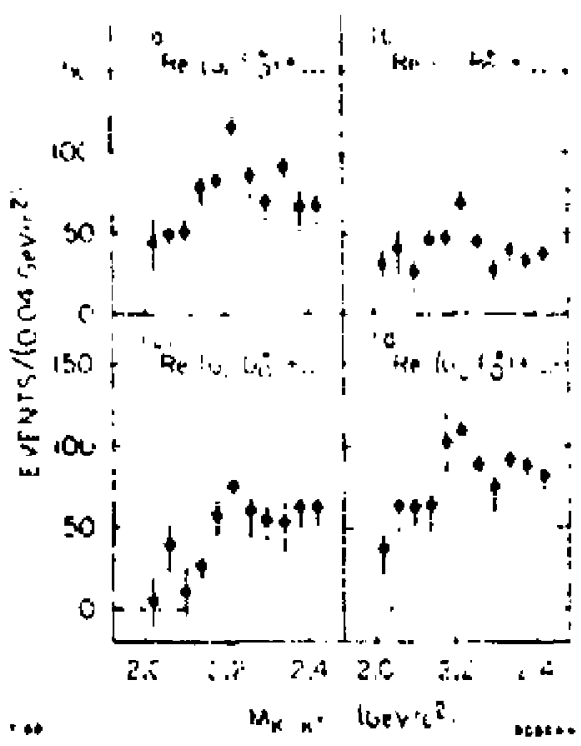

Fig. 5. Moments sums from reaction (1) dominated by leading $\mathrm{G}$ wave interference terms according to the model described in the text

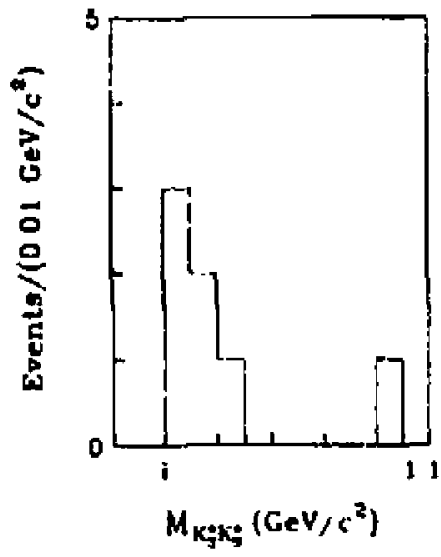

Fig. 6 The Kk threshold iegion oireacton (2)

\section{CONCLUSIONS}

The leading natural $\mathrm{J}^{\mathrm{f}}$ nonets up to $4^{+}$all now have good candidates for the Ir mainly s̄ members; in each case the masses are consistent with magic mixing. There are also now two strong candidates to be the strangeonitum members of the $\mathrm{I}^{+}$nonets, 


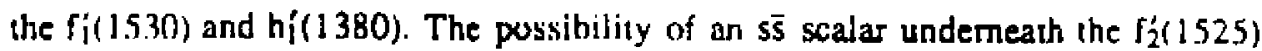
suggests a nomnal pattern of mass splitings for this nonet also. However, there are too many $0^{+}$mesons! The $X(2220)$ may well be a conventional $\mathbf{s} \bar{s}$ state, but there is strong evidence that the $f_{1}(1420), \eta(1440)$ and $f_{2}(1720)$ are not.

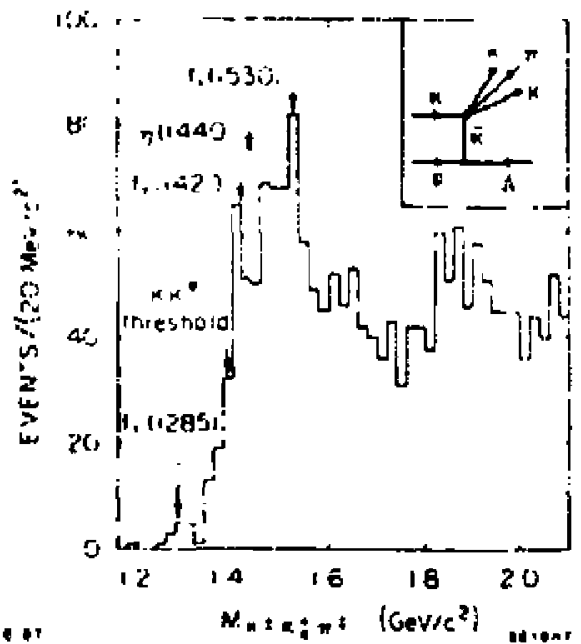

Fig. 7. The $\overline{\mathrm{K}} K \pi$ mass spectrum from reactions ( 3 ).

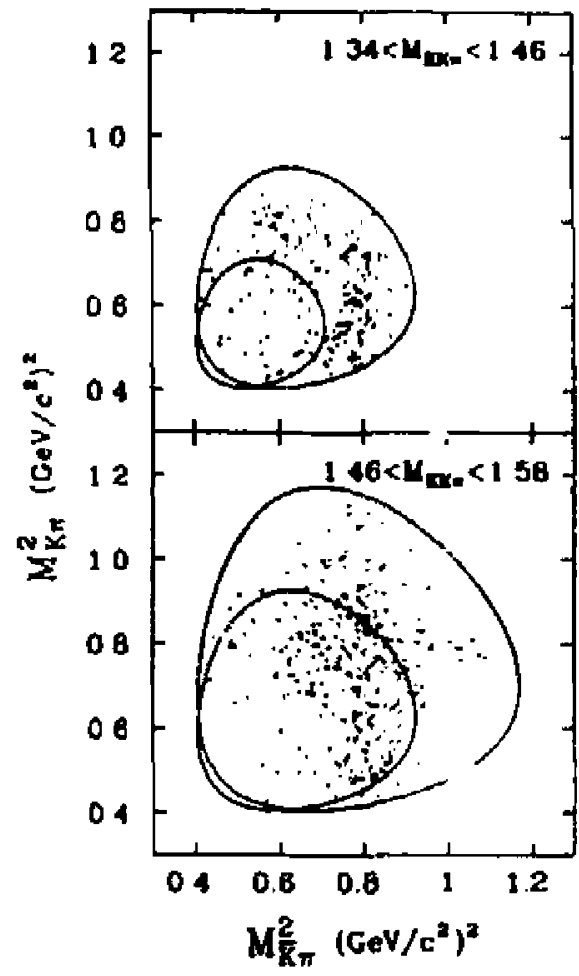

Fig. 8. $\overline{\mathrm{K}} \mathrm{K} \pi$ Daliz ploss from reactions (3) neat $K * K$ threshold.

\section{REFERENCES}

1. Particle Data Group, Phys. Lett. 170B, (1986).

2. D. Aston et al., The LASS Spectrometer, SL_AC-REP-298, (1986)

3. D. Aston e: al., Phys. Letr. 208B. 324, (1988).

4. D. Aston el al., DPNU-88-24 / SLAC-PUB-466) (1988).

5. D. Aston et al., Nucl. Phys. B301, 525. (1988).

6. D. Aston et al.. Phys. Lett. 201B. 573. (1988).

7. Talk by B. Ratcliff elsewhere in these proceedings and SLAC-PUB-4709 (1988).

8. C. Evangelista et al., Nucl. Phys. B 154, 381، (1979).

9. R. M. Baltrusaitis et al., Phys. Rev. Lell., 56, 107, (1986).

10. K. L. Au, D. Morgan and M. R. Pennirgton, Phys. Rev. D35. 1633, (1987).

11 Ph. Gavillet el al. Z. Phys. C16, 119. (1982). 